\title{
Butterfly distribution and habitat conservation status at A Rocha Dakatcha Nature Reserve, Kilifi County, Kenya
}

\author{
Beta, J. O. ${ }^{1 *}$, Ngaruiya G. ${ }^{1}$ \\ ${ }^{\mathbf{1} * \mathbf{1}}$ School of Pure and Applied Sciences, Department of Plant Sciences, Kenyatta \\ University, P. O. Box 43844-00100, Nairobi, Kenya. \\ ${ }^{1 *}$ Corresponding author's email: betajulius46@gmail.com
}

Received: 10 December 2020 / Accepted: 11 May 2021 / Published online: 30th June 2021

\begin{abstract}
Diverse human pressures are degrading coastal forests with profound implications on invertebrate biodiversity. Butterfly species are key ecosystem indicators and their distribution may become a campaign tool towards conservation of specific habitats. However, a baseline survey of Dakatcha butterflies is long overdue, necessitating this study at A Rocha Dakatcha Nature Reserve (ARDNR) in Kilifi County. The objective was to identify Dakatcha butterfly species, their distribution, threats to the forest and conservation measures by the community in 2019. The modified pollard walk method was used to collect butterfly species from 21 transects from which the Shannon index of diversity, Margalef's species richness index and evenness index were calculated. A total of 125 butterflies from five distinct vegetation types were captured and identified to represent 42 species and 25 genera from the documented 5 butterfly families in Kenya. Questionnaires were administered to twenty-nine community members on threats and conservation activities in ARDNR. The exercise confirmed that main threats to ARDNR butterfly habitat are agriculture, charcoal production from Diospyros corni and Dobera glabra and timber extraction from Manilkara mochisia, Brachystegia spiciformis, Thespesia danis and Brachylaena huillensis trees. The targeted deforestation of key tree species providing a suitable microclimate and an array of nectar sources to all threaten butterfly existence in the forest. Fortunately, there are five main stakeholders implementing diverse conservation projects including promotion of energy-saving jikos and beekeeping. In conclusion this study confirms existence of all five families of Kenyan butterflies in ARDNR. The presence of 13 butterfly species in the regenerating forest patch further
\end{abstract}

affirming the importance of practical all-inclusive forest management and that community awareness enhances diversification of livelihood activities alongside sustainable forest utilization.

Keywords: Butterfly diversity, abundance, protected areas, community groups, forest conservation.

\section{INTRODUCTION}

Dakatcha forest also referred as the Dakatcha woodland covers an area of 465,070 acres of land with 32\% being forest and $17 \%$ is woodland (Birdlife, 2021). Dakatcha woodland is one of the last patches of the relatively intact coastal woodland that together with the Arabuko Sokoke F Forest (ASF) and the fragments of Madunguni forest form the only remaining part of the Northernmost block of Miombo woodland that used to extend from southern Somalia to Northern Mozambique (Musila et al. 2006). The woodland is recognized by Conservation International as a coastal forests global hotspot and a Key Biodiversity Area (KBA) (Mbuvi et al. 2011). Unfortunately, 50\% of this important indigenous woodland has been degraded by agricultural expansion, unregulated logging, rampant charcoal burning and the expansion of commercial pineapple plantations (A Rocha, 2020). Such rapid and great loss has already given rise to a fragmented coastal forest forcing stakeholders to institute a holistic sustainable forest management plan to ensure a "win-win" scenario for the community and biodiversity (Nature Kenya, 2014).

To further counter the rapid degradation of this important ecosystem, the local community has established eight 
Community Conserved Areas (CCAs) from 2014 and the government has gazetted six Kaya forests (Nature Kenya, 2014). Notably, a private reserve of 2100 acres by A Rocha Kenya is galvanizing restoration of the forest through their Dakatcha Nature Reserve (ARDNR) established in 2014. The primary objectives of the community-led initiative is to conserve the indigenous coastal forest and its threatened wildlife particularly endemic species such as sokoke scops owl (Otus ireneae), sokoke pipit (Anthus sokokensis), clarke's weaver (Ploceus golandi) and the golden-rumped elephantshrew (Rhynchocyon chrysopygus) (A Rocha, 2020). Such commendable actions necessitate regular updates of the local and regional biodiversity databases to a determine efficacy of small but connected in-situ conservation projects.

Butterflies are seen as good indicators of environmental changes mainly because of their specificity to vegetation type, rapid response to perturbation, taxonomic tractability and ease of sampling (Gowda et al, 2011). Hence, robust butterfly population and diversity affirms the health status of a given forest ecosystem (Manzoor \& Sadat, 2013). Interestingly, Vu (2009) states that butterfly diversity is usually lower in natural forests, higher in disturbed forests and highest in moderately disturbed forests or forest edges. Unfortunately, this aspect has not been confirmed from the many studies on coastal forest because most of the studies focus on mammal, reptile and vascular plants populations due to their influence on the tourism sector. These haphazard studies leave gaps in invertebrate database especially butterfly species despite their importance for monitoring impacts of habitat degradation (Lindenmayer and Likens, 2009) and environmental change on biodiversity (Bonebrake et al. 2010). Though neighbouring Arabuko Sokoke Forest has had several butterfly surveys including those done by Ayiemba (1997) and Aden and Dharani (2019), Dakatcha still lacks a comprehensive butterfly database. Hence, this paper seeks to contribute to the coastal butterfly database as an urgent aspect considering that degradation of natural forest habitats is a conservation priority (Birdlife, 2021).

Accordingly, two entwined knowledge gaps such as lack of an up-to-date butterfly species database and the role of the community in reducing forest degradation informed this study in Dakatcha woodland in 2019. The objectives were to analyse butterfly diversity across the vegetation types in ARDNR, establish anthropogenic threats towards forest resources and delineate activities promoting sustainable forest utilization by the community within the Dakatcha woodland.

\section{METHODOLOGY}

\section{Study area}

Dakatcha woodland is an unprotected forest patch comprising of different vegetation types and seasonal wetlands and which lies between latitude $2^{\circ} 52^{\prime} 05^{\prime \prime} S$ and longitude $39^{\circ} 56^{\prime} 59^{\prime \prime} \mathrm{E}$ in Kilifi County (Figure 1). This study was done at two sections within the woodland. First, the butterfly study was conducted at the A Rocha Kenya's Dakatcha Nature Reserve. Second, data on community threats and conservation activities were collected randomly from the households within the Dakatcha woodland area.

The mean annual rainfall of Dakatcha woodland is 500$900 \mathrm{~mm}$ that is distributed in two rain seasons: long rains from March to June while short rains from October to December. The area's mean annual temperature is $24^{\circ} \mathrm{C}$ to $32^{\circ} \mathrm{C}$ where the mean maximum temperature is $28^{\circ} \mathrm{C}$ to $31^{\circ} \mathrm{C}$ and the minimum temperature is $20^{\circ} \mathrm{C}$ to $23^{\circ} \mathrm{C}$ (Nature Kenya, 2014).

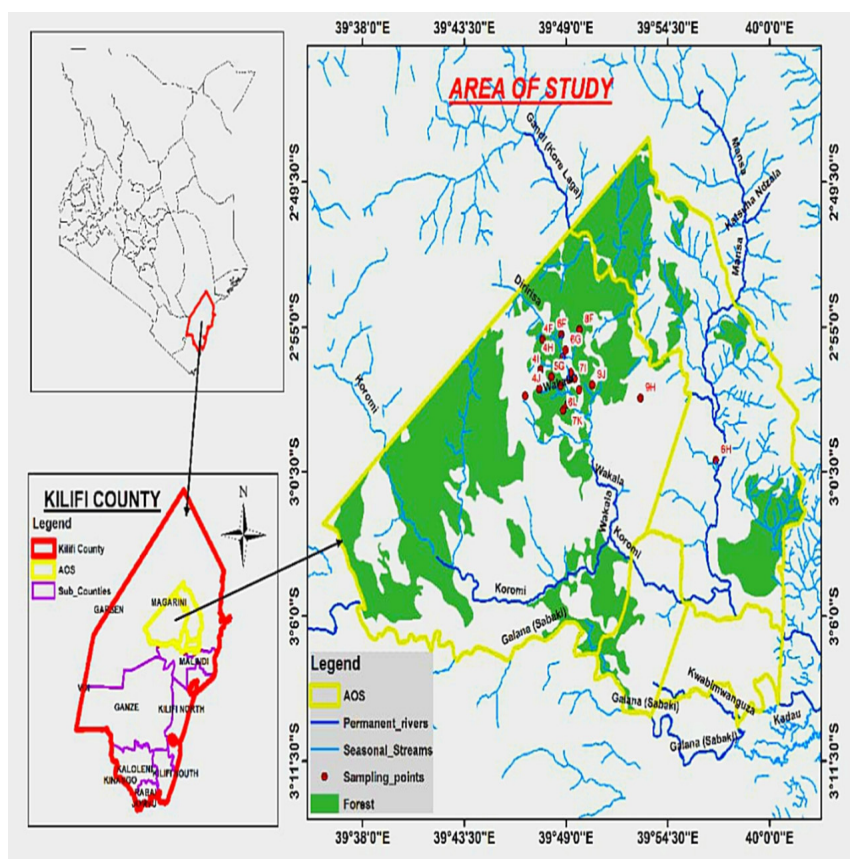

Figure 1: A map of Dakatcha woodland (Source: Author)

The area is mainly inhabited by the Mijikenda, specifically the Giriama community with an estimated population of 191610 in 2019 (KNBS, 2019). The population is sparsely distributed with more people being concentrated in major trading centres such as Marafa, Garashi, Baricho and Adu. The people are mainly involved in small scale crop production and animal husbandry. Recent interest in pineapple farming has increased logging and charcoal burning to create land for the pineapple farms (A Rocha, 2020 \& Nature Kenya, 2014). 


\section{Study Design}

\section{Butterfly distribution study}

Intensive sampling of butterflies was carried out in June and December 2019. Butterflies were studied using the modified pollard walk method (Pollard, 1977) which involved modified belt transects of approximately 20 square meters wide and $1 \mathrm{~km}$ long. Transects were located along paths and small roads in all the vegetation types of the woodland. Butterflies were captured between 7am $6 \mathrm{pm}$ using a butterfly net when either in flight or when settled and secured by a rapid sweep of the net. Thereafter, the butterfly net was held upside down and the captured butterfly samples collected carefully after stunning the butterfly by pinching their thorax.

Three pictures of both the lower and the upper parts of the captured butterfly wings were taken from each specimen. This was carefully done by folding the butterfly wings backward to avoid losing the colourful scales from the wings that aid in identification. The pictures were then uploaded to the virtual museum of FitzPatrick Institute of Ornithology for identification purposes. Additional data recorded included the date, time, locality of capture, coordinates of the area and the collector's name. Specimens were then mounted, labelled and preserved in the Lepidoptera collection at National Museums of Kenya.

\section{Threats and community participation in conservation of ARDNR}

Questionnaires were administered to 29 persons identified using random sampling to identify threats to the forest habitat and the conservation activities in the ARDNR area. The focus was on to find out source of conservation knowledge, participation level and the conservation activities in the Dakatcha community. In addition, activities going on in the woodland were recorded and pictures taken for further inquiry from the community.

\section{Data Interpretation and Analysis}

Butterfly data were pooled for total diversity and calculated using the formulas below.

1. Margalef's species richness index was used to compare species richness across different habitats.

$$
D m g=\frac{s-1}{\log N} \quad \begin{aligned}
& \text { Where, } \\
& \mathrm{S} \text { is the number of species. } \\
& \mathrm{N} \text { is the sampling size. }
\end{aligned}
$$

2. Shannon index of diversity was used to calculate the diversity of butterfly species across habitat
Where,

$$
\begin{aligned}
H^{\prime}=\frac{N \ln N-\sum(n i \ln n i)}{N} & \begin{array}{l}
N \text { is the total number } \\
\text { of observed species. } \\
n \text { i is the number of } \\
\text { individuals in species } i .
\end{array}
\end{aligned}
$$

3. The evenness index was used to calculate whether species are distributed evenly across different habitats.

$$
E_{D}=\frac{H^{\prime}}{\ln N} \quad \begin{aligned}
& \begin{array}{l}
\text { Where, } \\
\text { in is proportion of maximum value } \\
\text { of individuals. }
\end{array}
\end{aligned}
$$

\section{RESULTS AND DISCUSSION}

\section{Butterfly Diversity}

A total of 42 species of butterfly were recorded belonging to 25 genera and the key five families including Papilionidae, Pieridae, Nymphalidae, Lycaenidae and Hesperidae. ARDNR hosts family Hesperiidae consisted of one genus and one species $(0.8 \%)$, Papilionidae represented by two genera and four species (4.80\%), Lycaenidae with two genera and three species (5.0\%), Nymphalidae with 11 genera and 15 species (36\%) and Pieridae with nine genera and 19 species (52.80\%) (Figure 2).

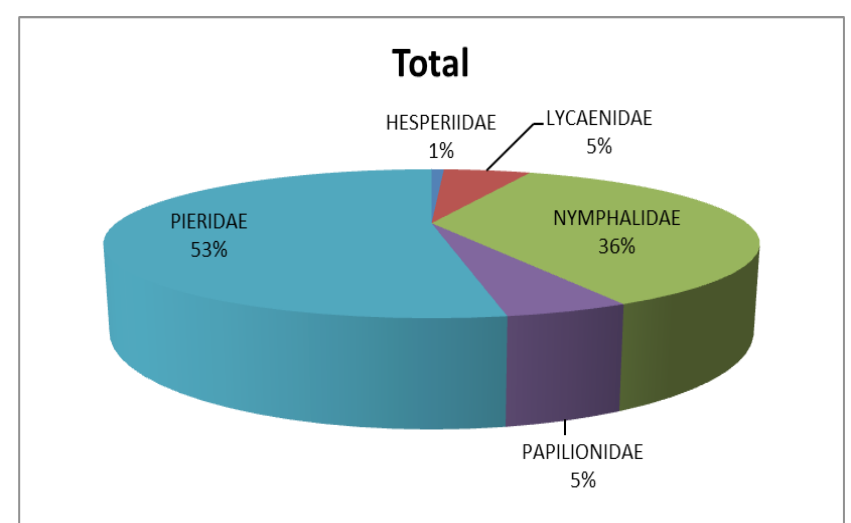

Figure 2: Family-wise dominance of butterfly species from ARKDNR habitat

The total captured species represent about 14\% of butterfly species recorded at the nearby Arabuko Sokoke Forest which is 45 times larger than ARDNR and hosts about 301 butterfly species (Ayiemba, 1997). A recent study conducted by Aden \& Dharani (2019) in Arabuko Sokoke Forest Nature Reserve recorded 106 species from 49 Genera of five families Papilionidae, Pieridae, Nymphalidae, Lycaenidae and Hesperidae.

Notably, the rainy season over the December holiday could also be a reason for few members of family Hesperiidae (Figure 2) being recorded as some might have migrated to a drier areas or rain might have an influence on their breeding behaviour. Ayiemba (1997) indicated that this 
genus could have more representation in coastal forests.

Butterflies from the family Papilionidae were sighted to move at fast rates and settles on flowers on tall trees thus only a few individuals were captured. Moreover, sweep nets could be biased towards the larger butterflies that are more easily identified either in flight or in traps.

In terms of evenness index the main five vegetation types had a medium diversity i.e., $1.5<\mathrm{X}<2.5$ except for the mixed forest that recorded a high diversity of 3.204 with the evenness E" of 0.9421 (Table 1). The habitats indicated in table 1 were restricted to only those in which butterfly species were captured during the survey, therefore, this does not mean that their ranges were only confined to these habitats.

The results confirm the richness of ARDNR because it supports all five families Papilionidae, Nymphalinidae, Pieridae, Lycaenidae and Hesperidae that have ever been documented in Kenyan coastal forests by Larsen (1991), Ayiemba (1997) and Aden \& Dharani (2019).

\section{Butterfly habitat distribution}

A total of 125 butterflies were captured in 21 transects from five distinct vegetation types of Brachystegia, Cynometra, mixed forest, recently cultivated land (mixed herbs) and grassland. The comprehensive list in Table 2 below will allow the readers to view online pictures and ecological details of captured butterfly species by clicking on the provided link from the virtual museum database.

The highest captured butterfly numbers were from the mixed forest and the mixed herbs (regenerating) habitats (Figure 3). The high numbers at the mixed forest seem to be supported by the open nature and the stratification of the vegetation which enables flowers with enough nectar sources and provides larval host plants for the larvae. Such habitat attracts both the specialist and generalist species and the savanna species as well, the latter being mostly the Pieridae. This finding is consistent with Habel et al. (2018) who stated that different habitats in each area contribute more to butterfly species diversity.

Table 1: Summary of the butterfly statistics at ARDNR

\begin{tabular}{|l|c|c|c|}
\hline \multicolumn{1}{|c|}{ Site } & Margalef richness index & Shannon Index & Evenness (E”) \\
\hline Brachystegia forest & 3.789 & 2.305 & 0.9611 \\
\hline Cynometra forest & 3.04 & 2.025 & 0.974 \\
\hline Mixed forest & 7.112 & 3.204 & 0.9421 \\
\hline Mixed herbs (recently cultivated) & 3.432 & 2.399 & 0.9352 \\
\hline Tall grass & 1.82 & 1.427 & 0.8867 \\
\hline
\end{tabular}

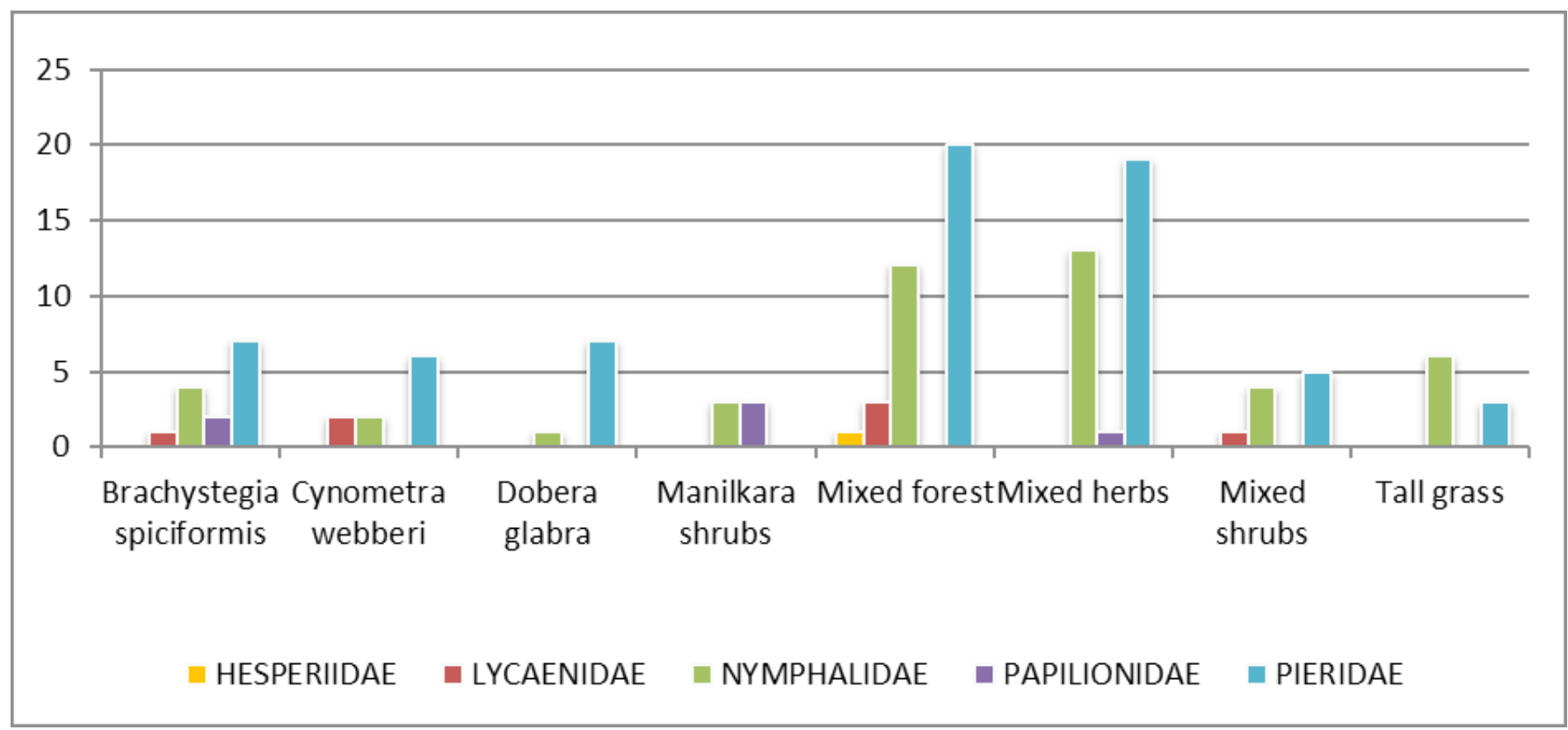

Figure 3: Distribution of butterfly families in Dakatcha woodland 
Table 2: A comprehensive list of captured butterfly species in ARDNR

\begin{tabular}{|c|c|c|c|c|}
\hline No. & Family & species name & $\begin{array}{l}\text { Vegetation } \\
\text { zonations }\end{array}$ & VMLink \\
\hline 1 & HESPERIIDAE & Spialia $s p$ & Mixed forest & http://vmus.adu.org.za/?vm=LepiMAP-689377 \\
\hline 2 & \multirow{7}{*}{ LYCAENIDAE } & Hypolycaena pachalica & Mixed forest & http://vmus.adu.org.za/?vm=LepiMAP-689578 \\
\hline 3 & & Hypolycaena pachalica & Mixed forest & http://vmus.adu.org.za/?vm=LepiMAP-689582 \\
\hline 4 & & Leptotes sp & Cynometra webberi & http://vmus.adu.org.za/?vm=LepiMAP-689595 \\
\hline 5 & & $\begin{array}{l}\text { Hypolycaena philippus } \\
\text { philippus }\end{array}$ & Mixed forest & http://vmus.adu.org.za/?vm=LepiMAP-689798 \\
\hline 6 & & Hypolycaena pachalica & $\begin{array}{l}\text { Brachystegia } \\
\text { spiciformis }\end{array}$ & http://vmus.adu.org.za/?vm=LepiMAP-690374 \\
\hline 7 & & Leptotes sp & Mixed forest & http://vmus.adu.org.za/?vm=LepiMAP-701099 \\
\hline 8 & & Leptotes sp & Cynometra webberi & http://vmus.adu.org.za/?vm=LepiMAP-701109 \\
\hline 9 & \multirow{45}{*}{ NYMPHALIDAE } & Hamanumida daedalus & \multirow{12}{*}{ Mixed forest } & http://vmus.adu.org.za/?vm=LepiMAP-689379 \\
\hline 10 & & Acraea anemosa & & http://vmus.adu.org.za/?vm=LepiMAP-689381 \\
\hline 11 & & Danaus chrysippus dorippus & & http://vmus.adu.org.za/?vm=LepiMAP-689382 \\
\hline 12 & & Acraea anemosa & & http://vmus.adu.org.za/?vm=LepiMAP-689383 \\
\hline 13 & & Acraea anemosa & & http://vmus.adu.org.za/?vm=LepiMAP-689388 \\
\hline 14 & & Byblia anvatara acheloia & & http://vmus.adu.org.za/?vm=LepiMAP-689575 \\
\hline 15 & & Junonia natalica natalica & & http://vmus.adu.org.za/?vm=LepiMAP-689583 \\
\hline 16 & & Acraea neobule neobule & & http://vmus.adu.org.za/?vm=LepiMAP-689589 \\
\hline 17 & & Ypthima sp & & http://vmus.adu.org.za/?vm=LepiMAP-689598 \\
\hline 18 & & Byblia ilithyia & & http://vmus.adu.org.za/?vm=LepiMAP-689600 \\
\hline 19 & & Byblia anvatara acheloia & & http://vmus.adu.org.za/?vm=LepiMAP-689603 \\
\hline 20 & & Byblia anvatara acheloia & & http://vmus.adu.org.za/?vm=LepiMAP-689604 \\
\hline 21 & & Danaus chrysippus dorippus & \multirow{13}{*}{$\begin{array}{l}\text { Mixed herbs (recently } \\
\text { cultivated) }\end{array}$} & http://vmus.adu.org.za/?vm=LepiMAP-689799 \\
\hline 22 & & Danaus chrysippus dorippus & & http://vmus.adu.org.za/?vm=LepiMAP-689800 \\
\hline 23 & & Acraea neobule neobule & & http://vmus.adu.org.za/?vm=LepiMAP-689801 \\
\hline 24 & & Phalanta phalantha aethiopica & & http://vmus.adu.org.za/?vm=LepiMAP-689808 \\
\hline 25 & & Acraea neobule neobule & & http://vmus.adu.org.za/?vm=LepiMAP-689809 \\
\hline 26 & & Junonia oenone oenone & & http://vmus.adu.org.za/?vm=LepiMAP-689863 \\
\hline 27 & & Junonia oenone oenone & & http://vmus.adu.org.za/?vm=LepiMAP-689864 \\
\hline 28 & & Danaus chrysippus dorippus & & http://vmus.adu.org.za/?vm=LepiMAP-689869 \\
\hline 29 & & Acraea neobule neobule & & http://vmus.adu.org.za/?vm=LepiMAP-689870 \\
\hline 30 & & Hypolimnas misippus & & http://vmus.adu.org.za/?vm=LepiMAP-689871 \\
\hline 31 & & Acraea neobule neobule & & http://vmus.adu.org.za/?vm=LepiMAP-689873 \\
\hline 32 & & Hypolimnas misippus & & http://vmus.adu.org.za/?vm=LepiMAP-689882 \\
\hline 33 & & Junonia hierta cebrene & & http://vmus.adu.org.za/?vm=LepiMAP-689884 \\
\hline 34 & & Phalanta phalantha aethiopica & Manilkara shrubs & http://vmus.adu.org.za/?vm=LepiMAP-689885 \\
\hline 35 & & Acraea anemosa & Manilkara shrubs & http://vmus.adu.org.za/?vm=LepiMAP-689967 \\
\hline 36 & & Hypolimnas misippus & Manilkara shrubs & http://vmus.adu.org.za/?vm=LepiMAP-689969 \\
\hline 37 & & Byblia anvatara acheloia & Dobera glabra & http://vmus.adu.org.za/?vm=LepiMAP-689973 \\
\hline 38 & & Junonia natalica natalica & Mixed forest & http://vmus.adu.org.za/?vm=LepiMAP-690376 \\
\hline 39 & & Byblia ilithyia & Tall grass & http://vmus.adu.org.za/?vm=LepiMAP-701086 \\
\hline 40 & & Byblia ilithyia & Tall grass & http://vmus.adu.org.za/?vm=LepiMAP-701087 \\
\hline 41 & & Byblia ilithyia & Tall grass & http://vmus.adu.org.za/?vm=LepiMAP-701090 \\
\hline 42 & & Junonia natalica natalica & Tall grass & http://vmus.adu.org.za/?vm=LepiMAP-701091 \\
\hline 43 & & Junonia natalica natalica & Tall grass & http://vmus.adu.org.za/?vm=LepiMAP-701092 \\
\hline 44 & & Byblia ilithyia & Tall grass & http://vmus.adu.org.za/?vm=LepiMAP-701094 \\
\hline 45 & & Byblia anvatara acheloia & Mixed forest & http://vmus.adu.org.za/?vm=LepiMAP-701096 \\
\hline 46 & & Byblia ilithyia & Mixed forest & http://vmus.adu.org.za/?vm=LepiMAP-701098 \\
\hline 47 & & Byblia ilithyia & Mixed forest & http://vmus.adu.org.za/?vm=LepiMAP-701100 \\
\hline 48 & & Eurytela dryope angulata & Cynometra webberi & http://vmus.adu.org.za/?vm=LepiMAP-701103 \\
\hline 49 & & Byblia anvatara acheloia & Cynometra webberi & http://vmus.adu.org.za/?vm=LepiMAP-701104 \\
\hline 50 & & Telchinia serena & $\begin{array}{l}\text { Brachystegia } \\
\text { spiciformis }\end{array}$ & http://vmus.adu.org.za/?vm=LepiMAP-701187 \\
\hline 51 & & Byblia ilithyia & $\begin{array}{l}\text { Brachystegia } \\
\text { spiciformis }\end{array}$ & http://vmus.adu.org.za/?vm=LepiMAP-701188 \\
\hline 52 & & Byblia anvatara acheloia & $\begin{array}{l}\text { Brachystegia } \\
\text { spiciformis }\end{array}$ & http://vmus.adu.org.za/?vm=LepiMAP-701189 \\
\hline 53 & & Melanitis leda & $\begin{array}{l}\text { Brachystegia } \\
\text { spiciformis }\end{array}$ & http://vmus.adu.org.za/?vm=LepiMAP-701195 \\
\hline
\end{tabular}




\begin{tabular}{|c|c|c|c|c|}
\hline 54 & \multirow{6}{*}{ PAPILIONIDAE } & Papilio demodocus demodocus & $\begin{array}{l}\text { Mixed herbs (recently } \\
\text { cultivated) }\end{array}$ & http://vmus.adu.org.za/?vm=LepiMAP-689875 \\
\hline 55 & & Graphium philonoe philonoe & Manilkara shrubs & http://vmus.adu.org.za/?vm=LepiMAP-689968 \\
\hline 56 & & Papilio demodocus demodocus & Manilkara shrubs & http://vmus.adu.org.za/?vm=LepiMAP-689970 \\
\hline 57 & & Graphium philonoe philonoe & Manilkara shrubs & http://vmus.adu.org.za/?vm=LepiMAP-689971 \\
\hline 58 & & Graphium colonna & $\begin{array}{c}\text { Brachystegia } \\
\text { spiciformis }\end{array}$ & http://vmus.adu.org.za/?vm=LepiMAP-690373 \\
\hline 59 & & $\begin{array}{l}\text { Papilio constantinus } \\
\text { constantinus }\end{array}$ & $\begin{array}{c}\text { Brachystegia } \\
\text { spiciformis }\end{array}$ & http://vmus.adu.org.za/?vm=LepiMAP-701192 \\
\hline 60 & \multirow{66}{*}{ PIERIDAE } & Colotis evagore antigone & Mixed forest & http://vmus.adu.org.za/?vm=LepiMAP-689378 \\
\hline 61 & & Colotis calais calais & Mixed forest & http://vmus.adu.org.za/?vm=LepiMAP-689389 \\
\hline 62 & & Dixeia doxo costata & Mixed forest & http://vmus.adu.org.za/?vm=LepiMAP-689390 \\
\hline 63 & & Belenois gidica abyssinica & Mixed forest & http://vmus.adu.org.za/?vm=LepiMAP-689574 \\
\hline 64 & & Nepheronia buquetii buquetii & Mixed forest & http://vmus.adu.org.za/?vm=LepiMAP-689576 \\
\hline 65 & & Nepheronia buquetii buquetii & Mixed forest & http://vmus.adu.org.za/?vm=LepiMAP-689577 \\
\hline 66 & & Colotis euippe omphale & Mixed forest & http://vmus.adu.org.za/?vm=LepiMAP-689579 \\
\hline 67 & & Colotis evagore antigone & Mixed forest & http://vmus.adu.org.za/?vm=LepiMAP-689581 \\
\hline 68 & & Colotis protomedia & Mixed forest & http://vmus.adu.org.za/?vm=LepiMAP-689584 \\
\hline 69 & & Nepheronia buquetii buquetii & Mixed forest & http://vmus.adu.org.za/?vm=LepiMAP-689585 \\
\hline 70 & & Nepheronia buquetii buquetii & Mixed forest & http://vmus.adu.org.za/?vm=LepiMAP-689586 \\
\hline 71 & & Colotis protomedia & Mixed forest & http://vmus.adu.org.za/?vm=LepiMAP-689587 \\
\hline 72 & & Nepheronia buquetii buquetii & Mixed forest & http://vmus.adu.org.za/?vm=LepiMAP-689588 \\
\hline 73 & & Teracolus eris eris & Mixed forest & http://vmus.adu.org.za/?vm=LepiMAP-689590 \\
\hline 74 & & Eurema brigitta brigitta & Mixed forest & http://vmus.adu.org.za/?vm=LepiMAP-689591 \\
\hline 75 & & Teracolus eris eris & Cynometra webberi & http://vmus.adu.org.za/?vm=LepiMAP-689592 \\
\hline 76 & & Colotis protomedia & Cynometra webberi & http://vmus.adu.org.za/?vm=LepiMAP-689593 \\
\hline 77 & & Colotis ione & Cynometra webberi & http://vmus.adu.org.za/?vm=LepiMAP-689594 \\
\hline 78 & & Eronia dilatata & Mixed forest & http://vmus.adu.org.za/?vm=LepiMAP-689596 \\
\hline 79 & & Colotis ione & Mixed forest & http://vmus.adu.org.za/?vm=LepiMAP-689597 \\
\hline 80 & & Dixeia doxo costata & Mixed forest & http://vmus.adu.org.za/?vm=LepiMAP-689599 \\
\hline 81 & & Dixeia doxo costata & Mixed forest & http://vmus.adu.org.za/?vm=LepiMAP-689601 \\
\hline 82 & & Colotis calais calais & Mixed forest & http://vmus.adu.org.za/?vm=LepiMAP-689602 \\
\hline 83 & & Colotis protomedia & \multirow{19}{*}{$\begin{array}{l}\text { Mixed herbs (recently } \\
\text { cultivated) }\end{array}$} & http://vmus.adu.org.za/?vm=LepiMAP-689802 \\
\hline 84 & & Catopsilia florella & & http://vmus.adu.org.za/?vm=LepiMAP-689803 \\
\hline 85 & & Catopsilia florella & & http://vmus.adu.org.za/?vm=LepiMAP-689806 \\
\hline 86 & & Colotis ione & & http://vmus.adu.org.za/?vm=LepiMAP-689807 \\
\hline 87 & & Catopsilia florella & & http://vmus.adu.org.za/?vm=LepiMAP-689810 \\
\hline 88 & & Teracolus eris eris & & http://vmus.adu.org.za/?vm=LepiMAP-689811 \\
\hline 89 & & Catopsilia florella & & http://vmus.adu.org.za/?vm=LepiMAP-689865 \\
\hline 90 & & Pinacopteryx eriphia eriphia & & http://vmus.adu.org.za/?vm=LepiMAP-689866 \\
\hline 91 & & Teracolus eris eris & & http://vmus.adu.org.za/?vm=LepiMAP-689867 \\
\hline 92 & & Colotis evagore antigone & & http://vmus.adu.org.za/?vm=LepiMAP-689868 \\
\hline 93 & & Colotis protomedia & & http://vmus.adu.org.za/?vm=LepiMAP-689872 \\
\hline 94 & & Colotis protomedia & & http://vmus.adu.org.za/?vm=LepiMAP-689874 \\
\hline 95 & & Colotis evagore antigone & & http://vmus.adu.org.za/?vm=LepiMAP-689876 \\
\hline 96 & & Catopsilia florella & & http://vmus.adu.org.za/?vm=LepiMAP-689877 \\
\hline 97 & & Colotis ione & & http://vmus.adu.org.za/?vm=LepiMAP-689878 \\
\hline 98 & & Catopsilia florella & & http://vmus.adu.org.za/?vm=LepiMAP-689879 \\
\hline 99 & & Colotis protomedia & & http://vmus.adu.org.za/?vm=LepiMAP-689880 \\
\hline 100 & & Teracolus eris eris & & http://vmus.adu.org.za/?vm=LepiMAP-689881 \\
\hline 101 & & Teracolus eris eris & & http://vmus.adu.org.za/?vm=LepiMAP-689883 \\
\hline 102 & & Eronia dilatata & Dobera glabra & http://vmus.adu.org.za/?vm=LepiMAP-689972 \\
\hline 103 & & Colotis ione & Dobera glabra & http://vmus.adu.org.za/?vm=LepiMAP-689974 \\
\hline 104 & & Eronia dilatata & Dobera glabra & http://vmus.adu.org.za/?vm=LepiMAP-689975 \\
\hline 105 & & Dixeia doxo parva & Dobera glabra & http://vmus.adu.org.za/?vm=LepiMAP-690366 \\
\hline 106 & & Eronia dilatata & Dobera glabra & http://vmus.adu.org.za/?vm=LepiMAP-690370 \\
\hline 107 & & Colotis ione & Dobera glabra & http://vmus.adu.org.za/?vm=LepiMAP-690372 \\
\hline 108 & & Eurema brigitta brigitta & Brachystegia spiciformis & http://vmus.adu.org.za/?vm=LepiMAP-690375 \\
\hline 109 & & Dixeia doxo costata & Mixed forest & http://vmus.adu.org.za/?vm=LepiMAP-690377 \\
\hline 110 & & Eronia dilatata & Mixed forest & http://vmus.adu.org.za/?vm=LepiMAP-690378 \\
\hline 111 & & Colotis euippe omphale & Mixed forest & http://vmus.adu.org.za/?vm=LepiMAP-690379 \\
\hline 112 & & Eurema hecabe solifera & Tall grass & http://vmus.adu.org.za/?vm=LepiMAP-701088 \\
\hline 113 & & Colotis protomedia & Tall grass & http://vmus.adu.org.za/?vm=LepiMAP-701089 \\
\hline 114 & & Colotis danae eupompe & Tall grass & http://vmus.adu.org.za/?vm=LepiMAP-701093 \\
\hline 115 & & Colotis evenina sipylus & Mixed forest & http://vmus.adu.org.za/?vm=LepiMAP-701095 \\
\hline 116 & & Belenois creona severina & Mixed forest & http://vmus.adu.org.za/?vm=LepiMAP-701097 \\
\hline 117 & & Belenois aurota & Cynometra webberi & http://vmus.adu.org.za/?vm=LepiMAP-701102 \\
\hline 118 & & Colotis protomedia & Cynometra webberi & http://vmus.adu.org.za/?vm=LepiMAP-701110 \\
\hline 119 & & Colotis danae eupompe & Cynometra webberi & http://vmus.adu.org.za/?vm=LepiMAP-701185 \\
\hline 120 & & Colotis euippe omphale & \multicolumn{2}{|c|}{ Brachystegia spiciformis http://vmus.adu.org.za/?vm=LepiMAP-701190 } \\
\hline 121 & & Eronia dilatata & \multicolumn{2}{|c|}{ Brachystegia spiciformis http://vmus.adu.org.za/?vm=LepiMAP-701191 } \\
\hline 122 & & Eronia dilatata & \multicolumn{2}{|c|}{ Brachystegia spiciformis http://vmus.adu.org.za/?vm=LepiMAP-701193 } \\
\hline 123 & & Eronia dilatata & \multicolumn{2}{|c|}{ Brachystegia spiciformis http://vmus.adu.org.za/?vm=LepiMAP-701194 } \\
\hline 124 & & Dixeia doxo costata & \multicolumn{2}{|c|}{ Brachystegia spiciformis http://vmus.adu.org.za/?vm=LepiMAP-701196 } \\
\hline 125 & & Dixeia doxo costata & \multicolumn{2}{|c|}{ Brachystegia spiciformis http://vmus.adu.org.za/?vm=LepiMAP-701197 } \\
\hline
\end{tabular}


In addition, regeneration in the cultivated land supports a high diversity of herb plants with flowers that provide assorted nectar sources for butterflies. This was confirmed by the 13 butterfly species observed in the mixed herbs area affirming the gradual success of conservation activities in the regenerating habitat. Thereby, the results conform to the study done by $\mathrm{Vu}$ (2009) showing that butterfly diversity is usually lower in natural forests, higher in disturbed forests, and highest in moderately disturbed forests or forest. Drawing on this understanding, forest managers can encourage the community to adopt the "Shamba System" or the Plantation Establishment and Livelihood Improvement Scheme (PELIS). PELIS which effectively supports biodiversity, livelihoods and forest regeneration (Agevi et al., 2016).

\section{Threats to butterfly habitat}

This objective was covered through detailed survey of the habitat in ARDNR and respondent feedback in the community. Deforestation was through charcoal burning (23\%) mainly targeting Diospyros cornii (mkulu) and Dobera glabra (mkami), illegal harvesting of poles (12\%), timber and carving wood (23\%) from Manilkara mochisia (Mnago), Brachystegia spiciformis (Mrihi), Thespesia danis (Muhowe) and Brachylaena huillensis (muhuhu) trees (Figure 4). Clearing of the understory for agriculture (35\%) especially pineapple plantations is responsible for the gradual reduction of Cynometra forests (mfunda). Fuelwood extraction (8\%) is the least threat to the butterfly habitat maybe due to the surveillance in the nature reserve.

The habitat threats can be perceived in two main ways. First, the targeted deforestation of key tree species that define a given specific forest habitat structure and these trees provide much needed canopy cover that encourages a conducive microclimate for butterflies. Second, the deliberate clearing of the forest understory herbs and shrubs for agriculture which then reduces available nectar sources for the butterflies.

\section{Community conservation efforts}

Respondents identified the following benefits from
Dakatcha forest as being the main reason they would participate in its conservation; food (23\%), employment $(6 \%)$, animal protein $(13 \%)$, honey $(21 \%)$, medicinal plants (11\%) and construction materials (25\%). Interestingly, some respondents were aware of the ecological services the forest provides to them such as attraction of rain $(62 \%)$ and soil erosion control (38\%) indicating that the community appreciates their forest habitat.

In terms of active conservation there are five main stakeholders driving the conservation agenda at ARDNR. The conservation projects revolve around community education and awareness on the destructive practices of charcoal burning and replacing unsustainable agriculture practices with conservation-led activities. These stakeholders are:

a) A Rocha Kenya community and environment education team working with local church organisations and schools.

b) Farming God's Way (FGW) organisation that actively trains farmers in conservation agriculture implementation to conserve Dakatcha forest ecosystem.

c) Nature Kenya and Birdlife organisations involved in conservation of the clarke's weaver bird (Ploceus golandi) and other endemic species.

d) Anglican church donating goat breeds as a source of milk and meat to the community and education on sustainable tomatoes farming.

e) Women groups like the Amani and Upendo groups constitute about $58 \%$ of those interviewed and they participate in farming of chicken, goats, and fast growing cassava hybrid (Tajirika). The groups also purchase energy saving jikos for its members.

Most of the training given to the community highlights the ecological interactions that enable them to harvest honey, medicinal plants and wild fruits. Such practical education encourages the community to participate in simple farming practices that earn income like beekeeping (23\%) and other options of clean energy (27\%) at the household level.

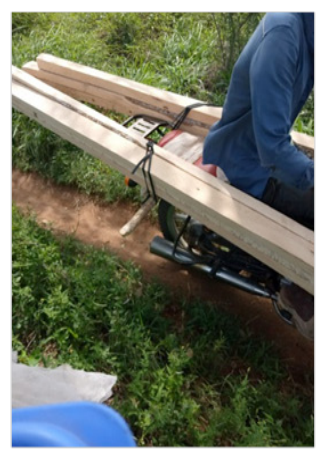

a. $\operatorname{Timber}(23 \%)$

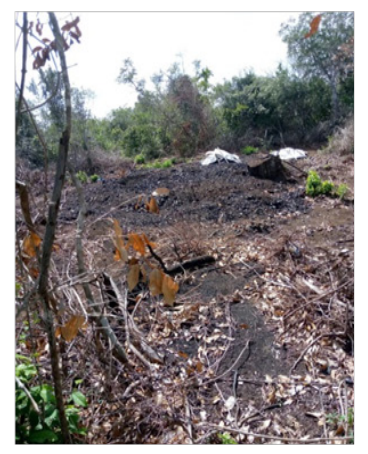

b. Charcoal $(23 \%)$

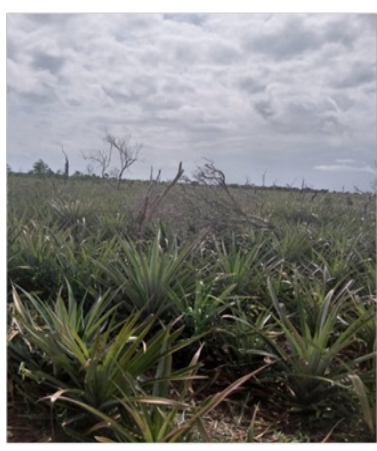

c. Pineapple plantation (35\%)

Figure 4: Deforestation activities degrading the indigenous habitat of ARDNR 


\section{CONCLUSION}

This study provides habitat specific baseline data of the butterflies in Dakatcha woodland. It has reaffirmed that the Dakatcha woodland harbours diverse species representing the five documented butterfly families in Kenya. This research has also shown that though butterflies are evenly distributed within four distinct habitats, they are in high numbers at the mixed forest habitat, a trend that conforms to other scientific studies. It also highlights threats to butterfly habitat through the deliberate illegal extraction of specific tree species and clearing of the understory for pineapple farming, which may impact butterfly distribution. On the positive side the study concludes by highlighting actions of key stakeholders to enhance awareness and increase participation towards sustainable utilization of the natural resources of the Dakatcha woodland. Such positive actions will no doubt increase availability of a large diversity of plants that enable existence of a diverse array of butterfly species that can attract tourists and researchers to the Dakatcha woodland and earn revenue for the community and Kilifi County. The study recommends further research on species abundance by analysing the correlation between species-habitat, species-vegetation and seasonal variation to guide implementation of proposed conservation strategies efficiently.

\section{ACKNOWLEDGMENT}

The study would not have been possible without the support from A Rocha Kenya staff particularly Dr. Colin Jackson for his mentorship, permission to conduct the study in the nature reserve and provision of the field equipment and to Mr. Samson Katisho for being an effective field guide and gracious host.

\section{REFERENCES}

A Rocha, (2020). A Rocha Dakatcha Nature Reserve. Creating a safe haven for five red listed species in the Dakatcha key biodiversity area, Kenya. Report by A Rocha Kenya. Available online at https://www. arocha.or.ke/wp-content/uploads/sites/16/2020/11/ Dakatcha_Conservation_Strategy.pdf on 19/04/2021

Aden, H. A. \&, Dharani, N. (2019). Investigation of the distribution and abundance of butterfly in the Nature Reserve of Arabuko Sokoke Forest Reserve, Kilifi County, Kenya. Int. J. Plant Anim. Environ. Sci, 9(4), 222-236. https://www.researchgate.net/ publication/338402265 Investigation of the Distribution and Abundance of Butterfly in the Nature Reserve of Arabuko Sokoke Forest Reserve Kilifi County Kenya.

Agevi, H., Mwendwa, K. A., Koros, H., Mulinya, C., Kawawa, R. C., Kimutai, D. K., Wabusya, M., Khanyufu, M., \& Jawuoro, S. (2016). PELIS forestry programme as a strategy for increasing forest cover and improving community livelihoods: case of Malava forest, western Kenya. American Journal of Agriculture and Forestry, 4(5),128-135. DOI:10.11648/j. ajaf.20160405.13.

Ayiemba, W. O. (1997). A study of the butterfly diversity in the Arabuko Sokoke Forest, Kenya. (Doctoral dissertation, University of Nairobi, Kenya). Retrieved from http://erepository.uonbi.ac.ke/bitstream/ handle/11295/24528/Ayiemba\%20_A\%20Study\%20 Of\% 20 The $\% 20$ Butterfly $\% 20$ Diversity $\% 20$ In $\% 20$ The $\% 20$ Arabuko-Sokoke $\% 20$ Forest $\% 2 c \% 20$ Kenya. pdf?sequence $=3$ \&isAllowed $=\mathrm{y}$.

Birdlife (2021). Important Bird Areas fact sheet: Dakatcha Woodland. BirdLife International. Downloaded from http://www.birdlife.org on 19/04/2021.

Bonebrake, T. C., Ponisio, L. C, Boggs, C. I., \& Ehrlich, P. R. (2010) More than just indicators: a review of tropical butterfly ecology and conservation. Biol. Conserv, 143, 1831-1841.

Gowda, R., Vijaya, K. H. T, Pramod, A. F., \& Hosetti, B. B. (2011). Butterfly diversity, seasonality and status in Lakkavalli range of Bhadra Wildlife Sanctuary, Karnataka. World Journal of Science and Technology, 1(11), 67-72.

Habel, J. C., Seibold, S., Ulrich, W., \& Schmitt T. (2018). Seasonality overrides differences in butterfly species composition between natural and anthropogenic forest habitats. Animal Conservation, 21(5), 405-413.

KNBS (2019). Kenya Population and Housing Census. A report by the Kenya National Bureau of Standards. Government of Kenya, Nairobi. Accessed from https:// www.knbs.or.ke/?wpdmpro=2019-kenya-populationand-housing-census-volume-i-population-by-countyand-sub-county on 19/04/2021

Lindenmayer, D. B., \& Likens G. E. (2009). Adaptive monitoring: A new paradigm for longterm research and monitoring. Trends Ecol. Evol, 4, 482-486.

Manzoor, F., \& Bin Sadat, H. (2013). Butterflies as indicator of climate change. Zoo's Print, 28, 19-22.

Mbuvi, M. T., Nahama, T., \& Musyoki, J. K. (2011). Master plan for Dakatcha woodland area: An important bird area. Draft. Nature Kenya. Marafa, Kenya. Pp. 2011-2035.

Musila, S. N., Ng'weno, F., Matiku, P., Mwema, M., Kanyanya, E., Mulwa, R., Musina, J., Buckley, P., \& S. Njehia (2006). Kenya's important bird areas, status and trends. Nature Kenya, Nairobi.

Nature Kenya (2014). Participatory management plan for Dakatcha woodland. Dakatcha Woodland Participatory Management Plan 2014-2019. Nature Kenya. Retrieved from https://issuu.com/nature kenya/ docs/dakatcha woodland participatory man on 
$19 / 04 / 2021$

Pollard, E. (1977). A method for assessing changes in the abundance of butterflies. Biological conservation, 12(2), 115-134.

Vu, L. V. (2009) Diversity and similarity of butterfly communities in five different habitat types at Tam Dao National Park, Vietnam. Journal of Zoology, 277(1), $15-22$. 